\title{
Frequency scaling and localization of geodesic acoustic modes in ASDEX Upgrade
}

\author{
G D Conway, C Tröster, B Scott, K Hallatschek and the \\ ASDEX Upgrade Team \\ ${ }^{1}$ Max-Planck-Institut für Plasmaphysik, EURATOM-Association IPP, D-85748 \\ Garching, Germany
}

\begin{abstract}
The frequency behaviour and localization of the geodesic acoustic mode $(\mathrm{GAM})$, believed to be a coherent plasma turbulence-generated $E_{\mathrm{r}} \times B_{\mathrm{T}}$ zonal flow oscillation, is studied in the ASDEX Upgrade tokamak using Doppler reflectometry. In typical elongated $(1.4<\kappa<1.75)$ plasmas with an X-point divertor configuration the GAM is observed only in the edge density gradient region $0.95<\rho_{\text {pol }}<1.0$ between the density pedestal top and the flux surface boundary. The GAM frequency $(5-25 \mathrm{kHz})$ is found to scale linearly as $\omega=G c_{\mathrm{S}} / R_{\mathrm{o}}$ (sound speed over major radius) but with an inverse dependence on the plasma elongation $\kappa$ and a weak direct dependence on the safety factor $q$. The lower the GAM frequency the more important it is expected to become in moderating the turbulence via shear decorrelation. A heuristic scaling law for the frequency scale factor $G \sim \mathcal{O}(1)$ involving $\kappa$ and finite aspect ratio $\epsilon$ terms has been obtained from dedicated parameter scans. For circular plasmas $\kappa \sim 1$ touching the limiter the density pedestal is weakened and the GAM is seen to reach in radially as far as $\rho_{\text {pol }} \sim 0.75$, depending on the $q$ profile, with a frequency scale $G \rightarrow \sqrt{2}$ consistent with theoretical predictions. Radially the GAM frequency is not a smooth function but displays a series of plateaus a few $\mathrm{cm}$ wide coinciding with peaks in the GAM amplitude, suggesting several zonal flow layers. At the plateau edges the GAM spectral peak splits into two frequency branches.
\end{abstract}

PACS numbers: 52.55.Fa, 52.35.Ra, 52.70.Gw

Submitted to: Plasma Phys. Control. Fusion 


\section{Introduction}

The current interest in zonal flows (ZF) and associated geodesic acoustic modes (GAMs) in magnetic confinement devices is motivated by their association with turbulence and plasma confinement. Analytic theory and numerical turbulence simulations, e.g. $[1,2]$, predicted the formation of static and oscillating $E_{\mathrm{r}} \times B$ poloidal plasma shear flows driven, not directly by the temperature and density gradients, but by non-linear interactions in gradient driven plasma turbulence (e.g. via Reynolds stress etc.) [3, 4]. These turbulence driven flows may in-turn moderate the turbulence, and hence affect the plasma transport, via either shear de-correlation (radial shearing of turbulence eddies which reduces the turbulence radial correlation length) or by acting as an energy sink $[5,6]$. Aside from sheared mean flows (non-oscillating, non-localized: $\omega=0, k_{\mathrm{r}}=0$ ), there are zonal flows (quasi-stationary but radially localized: $\omega \approx 0, k_{\mathrm{r}} \neq 0$ ) and oscillating flows at the geodesic acoustic frequency (a few $\mathrm{kHz})\left(\omega \neq 0, k_{\mathrm{r}} \neq 0\right)$ [7]. Both $\mathrm{ZF}$ and GAM flow perturbations have an axisymmetric $(m=n=0)$ mode structure but a finite radial extent $\left(k_{\mathrm{r}} a \gg 1\right)$, are essentially electrostatic (i.e. no strong magnetic component), and appear only on closed flux surfaces.

In the case of the GAM the flow perturbation couples via the geodesic curvature of the magnetic field to an axisymmetric pressure sideband mode $(m= \pm 1, n=0)$, the combination of which creates the eigenmode oscillation. Although the GAM is in general forced by the turbulence over a broad range of frequencies [8], it has a natural frequency, $\omega_{\text {GAM }}$. For a large aspect ratio, $R_{\mathrm{o}} \gg a$, circular plasma Winsor [9] derived the mode frequency:

$$
\omega_{\mathrm{GAM}}=2 \pi f_{\mathrm{GAM}}=\left(2+q^{-2}\right)^{1 / 2} c_{\mathrm{s}} / R_{\mathrm{o}},
$$

where $c_{\mathrm{s}}=\sqrt{\left(T_{\mathrm{e}}+\gamma_{\mathrm{i}} T_{\mathrm{i}}\right) / M_{\mathrm{i}}}$ is the ion sound speed and $R_{\mathrm{o}}$ is the plasma major radius. However, for non-circular plasmas experimental measurements show substantial deviations from this simple prediction. For example, initial measurement using Doppler reflectometry on the ASDEX Upgrade tokamak $\left(a / R_{\mathrm{o}}=0.5 / 1.65 \mathrm{~m}\right)$ show the GAM frequency scaling inversely with the plasma boundary elongation $\kappa_{b}$ and directly with the tokamak edge safety factor $q_{95}$ [11]. A similar decrease in $\omega_{\text {GAM }}$ with increasing $\kappa$ was observed on the DIII-D tokamak $\left(a / R_{\mathrm{o}}=0.6 / 1.7 \mathrm{~m}\right)$ using beam emission spectroscopy with $\omega_{\mathrm{GAM}} \sim c_{\mathrm{s}} / R_{\mathrm{o}}$, i.e. no $\sqrt{2}$ factor, but with an additional inverse dependence on $q[12]$. Other shaped machines, such as the JFT-2M tokamak $\left(a / R_{\mathrm{o}}=0.35 / 1.31 \mathrm{~m}\right)$ report $\omega_{\mathrm{GAM}} \sim c_{\mathrm{s}} / R_{\mathrm{o}}$ using heavy ion beam probes (HIBP) [14], while for Langmuir probes $\omega_{\mathrm{GAM}} \sim 2 c_{\mathrm{s}} / R_{\mathrm{o}}$ for $\kappa<1.7$ [15]. Conversely, for circular machines a scaling closer to Winsor's is seen: TEXT using HIBP [16], T-10 $\left(a / R_{\mathrm{o}}=0.3 / 1.5 \mathrm{~m}\right)$ using HIBP [17], TEXTOR $\left(a / R_{\mathrm{o}}=0.47 / 1.75 \mathrm{~m}\right)$ using reflectometry [18], HT-7 $\left(a / R_{\mathrm{o}}=\right.$ $0.27 / 1.22 \mathrm{~m}$ ) using probes (although with large scatter) [19], and the HL-2A tokamak $\left(a / R_{\mathrm{o}}=0.4 / 1.65 \mathrm{~m}\right)$ in divertor configuration with close to circular shape using probes [13]. Note for this comparison the same definition of $c_{\mathrm{s}}$ has been employed, where possible, with an ion specific heat ratio $\gamma_{i}=1$. 
It is important to understand the behaviour of the GAM frequency since the GAM may impact on the $E \times B$ shearing rate and hence turbulence reduction; providing its frequency is lower than the inverse turbulence decorrelation time $f_{\text {GAM }}<1 / \tau_{d}$ $[5,20,21,22,23]$. If the GAM frequency is reduced by plasma shaping then it may become more important to the effective shearing rate and thus in reducing the turbulence radial correlation length [20].

Most analytic formulations and modelling of the GAM properties have addressed large aspect ratio $R \gg a$ perfectly circular plasmas: e.g. fluid models [9, 10, 24, 25, 26] or drift kinetic equations [27]. The first detailed discussion of shaping effects appears in the work of Watari [22] which expands the kinetic drift equation to generic helical systems using a Fourier expansion of the magnetic field. With some simplifications to the dispersion relation the GAM frequency for a tokamak with a single dominant Fourier component can be shown to scale inversely with the $\kappa$. However, in general several Fourier components are required necessitating a numerical solution. Hallatschek, using a two-fluid approach, derives a frequency involving the ratio of two geometrical coefficients for the kinetic and compressional energy [23, 28]. For an elliptic Millerequilibrium in the high aspect ratio limit the coefficients can be expressed analytically, but, in general they must also be solved numerically. Nevertheless, indications of $\omega_{\mathrm{GAM}}$ decreasing with increasing $\kappa$ were found together with a sensitivity to the differential Shafranov shift $d R / d r[23]$.

GAM parameter dependence has also been investigated numerically using the gyrokinetic code ORB5 [29]. Here the effect of elongation appeared weak but stronger effects from triangularity $\delta$ and finite inverse aspect ratio $\epsilon=r / R$ were observed. Kendl, however, using a gyro-fluid electromagnetic model with drift wave turbulence and realistic (ie. experimental based) equilibria found a stronger $\kappa$ dependence for the frequency [30].

An experimental investigation of GAM frequency dependence is more complicated since the shape parameters are generally interrelated; for example, the triangularity tends to increase with elongation, along with the $q$ profile and magnetic shear etc. unless the plasma current is adjusted to compensate. Here, results from systematic parameter scans in the ASDEX Upgrade tokamak using Doppler reflectometry are presented. Based on the scan results and theory input several heuristic scaling models for the GAM frequency are formulated and tested against the full database of GAM measurements. A clear separation in the GAM frequency behaviour is found depending on the presence or absence of a strong density pedestal, e.g. large radial second derivatives $\partial^{2} / \partial r^{2}$ in the profiles. For a weak pedestal in a circular limiter plasma the GAM extends towards the core with a frequency scaling approaching the theoretical prediction, however, in the edge density gradient region the scaling becomes anomalous with an apparent $(1+\kappa)^{-1}$ dependence. Note that the detailed study presented here updates the preliminary experimental results on the GAM shape dependence reported in references [31,32]. Likewise, parameter effects on the GAM amplitude have also been observed (cf. [32]) but will be reported in more detail in a subsequent paper. 


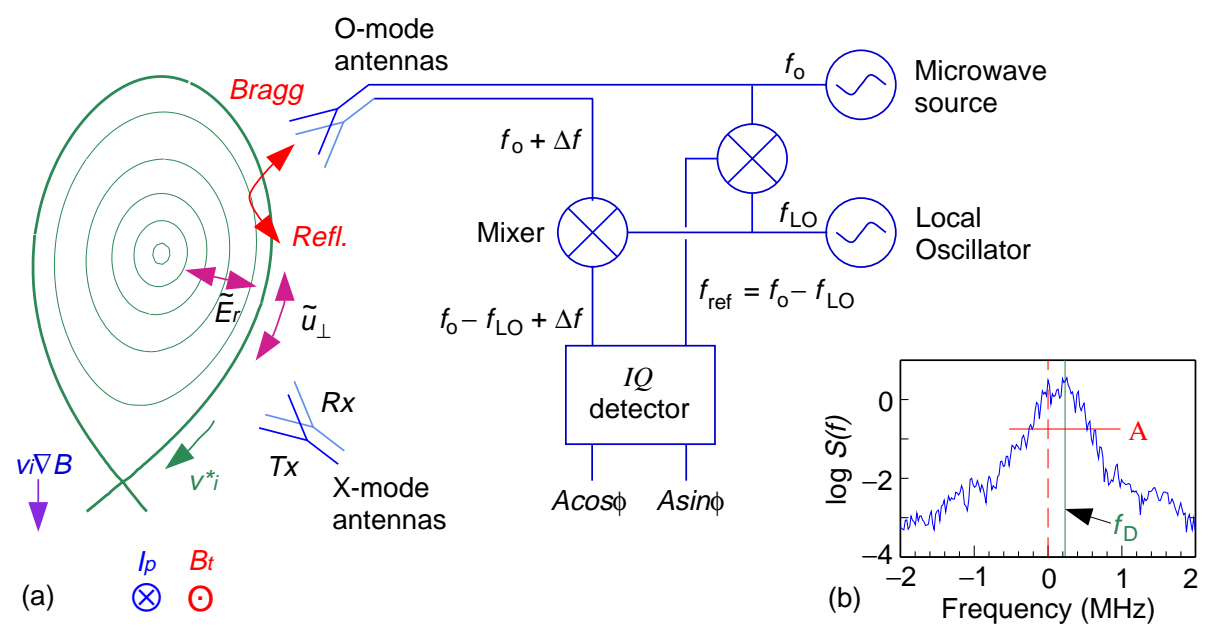

Figure 1. (a) Schematic of a Doppler reflectometer channel with tokamak poloidal plane showing positions of $\mathrm{O}$ and X-mode antenna pairs, and (b) example complex amplitude reflectometer spectrum from a typical ohmic discharge.

\section{Measurement technique}

The diagnostic approach is to measure the frequency and amplitude of coherent oscillations in the $E \times B$ plasma flow velocity using microwave Doppler reflectometry. Figure 1 shows a schematic of the diagnostic technique. A microwave beam is launched into the plasma with a deliberate tilt angle $\theta_{0}$ to the flux surface. As the beam propagates it is refracted and eventually reflected at the cutoff condition (when the

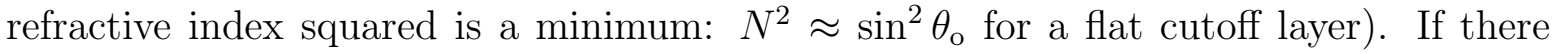
is sufficient turbulence at the cutoff region with a wavenumber satisfying the Bragg condition $k_{\perp}=2 k_{\mathrm{o}} N \approx 2 k_{\mathrm{o}} \sin \theta$ (where $k_{\mathrm{o}}$ is the microwave wavenumber) then a signal is back-scattered to the diagnostic receiver antenna. Further, any movement of the turbulence will generate a Doppler frequency shift, $f_{\mathrm{D}}=u_{\perp} k_{\perp} / 2 \pi$, in the received signal where $u_{\perp}=v_{E \times B}+v_{\text {phase }}$ is the perpendicular (to the static magnetic field) velocity of the turbulence moving in the plasma [33]. Since the measured velocity contains the $E \times B$ velocity, any fluctuations in $E_{\mathrm{r}}$ will appear directly as fluctuations in the Doppler shift frequency. In this technique the turbulence is essentially used as a tracer to access the flow perturbations. Coherent density fluctuations (MHD) can also appear in $f_{\mathrm{D}}$, but as they also modulate the backscattered signal amplitude $\mathcal{A}$ (a measure of $\tilde{n}_{e}$ at the selected $k_{\perp}$ ) they can be discriminated.

The diagnostic used on ASDEX Upgrade (AUG) consists of two heterodyne reflectometers with variable launch frequencies between $50-75 \mathrm{GHz}$ in $\mathrm{O}$ and $\mathrm{X}$-mode polarization [33]. The measurement location, which is obtained using a beam tracing code and spline fitted density profiles incorporating DCN interferometry, Thomson Scattering, Lithium-beam and FM profile reflectometry data, can be typically scanned from the plasma edge to around mid-radius on the tokamak low field side [34]. The antennas are fixed with typical tilt angles around $\theta_{\mathrm{o}} \sim \mathcal{O}\left(18^{\circ}\right)$ which translates to a 
(a)

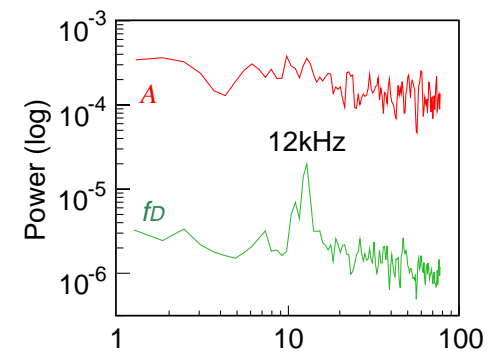

(b)

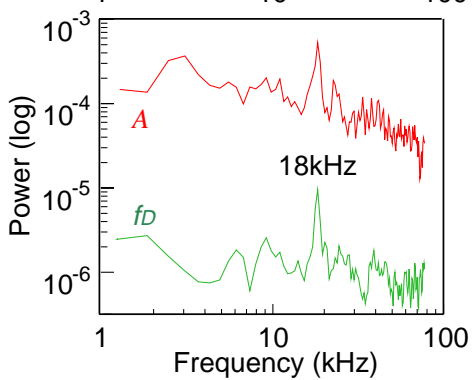

$\mathrm{Kb}=1.59$

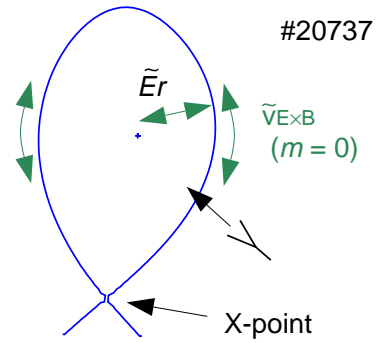

$\mathrm{Kb}=1.42$

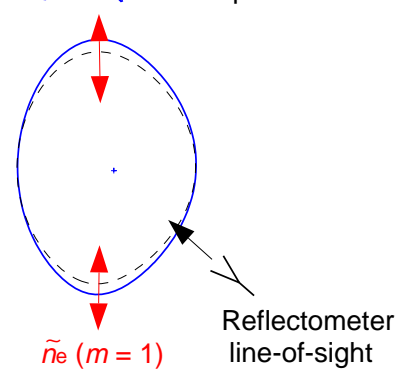

Figure 2. Edge Doppler frequency $f_{\mathrm{D}}$ and amplitude $\mathcal{A}$ spectra during (a) divertor $\kappa_{\mathrm{b}}=1.59$ and (b) inner limiter $\kappa_{\mathrm{b}}=1.42$ phases of $B_{T}=2 \mathrm{~T} / I_{p}=0.8 \mathrm{MA}$ ohmic discharge \#20737.

typical probed $k_{\perp} \sim 8 \mathrm{~cm}^{-1}$. In-phase $(I=A \cos \phi)$ and Quadrature $(Q=A \sin \phi)$ signals (allowing phase and amplitude separation) are sampled at $20 \mathrm{MHz}$, from which the complex amplitude spectrum $S(f)$ is computed. Figure 1(b) shows a typical example from an ohmic discharge. The Doppler shift is extracted from a weighted mean and the signal amplitude from the spectral integral. By sliding an FFT window (256 points) through the data stream a time sequence of $f_{\mathrm{D}}$ and $\mathcal{A}$ fluctuations can be generated. Full details of the analysis technique are given in [11].

\section{GAM features}

Nearly all ohmic and L-mode (neutral beam and electron cyclotron resonance) heated AUG discharges display large coherent oscillations or modes in the $f_{\mathrm{D}}$ spectra between $5-25 \mathrm{kHz}$ with an intensity of 1 to 2 orders of magnitude above the background. The oscillations appear even in the absence of MHD activity and are generally seen in the edge density gradient region of standard lower single-null diverted discharges - where the turbulence vorticity and $E_{\mathrm{r}}$ shear are largest [11]. No coherent activity is seen in the open-field SOL region $\left(f^{-1}\right.$ spectra), nor deep in the plasma core region (flat spectra). The mode has the features expected of a GAM, its frequency scales linearly with the ion sound speed $c_{\mathrm{s}}$ with no dependence on either the magnetic field $B$ or the mean plasma density $n_{\mathrm{e}}$, i.e. it has an acoustic nature. So far GAMs have not been observed in H-modes, possibly due to the lower turbulence level or higher rotational velocity shearing.

There is no measurable magnetic perturbation, and generally only a weak density perturbation. Since both the $\mathrm{O}$ and X-mode Doppler reflectometer antenna pairs are 

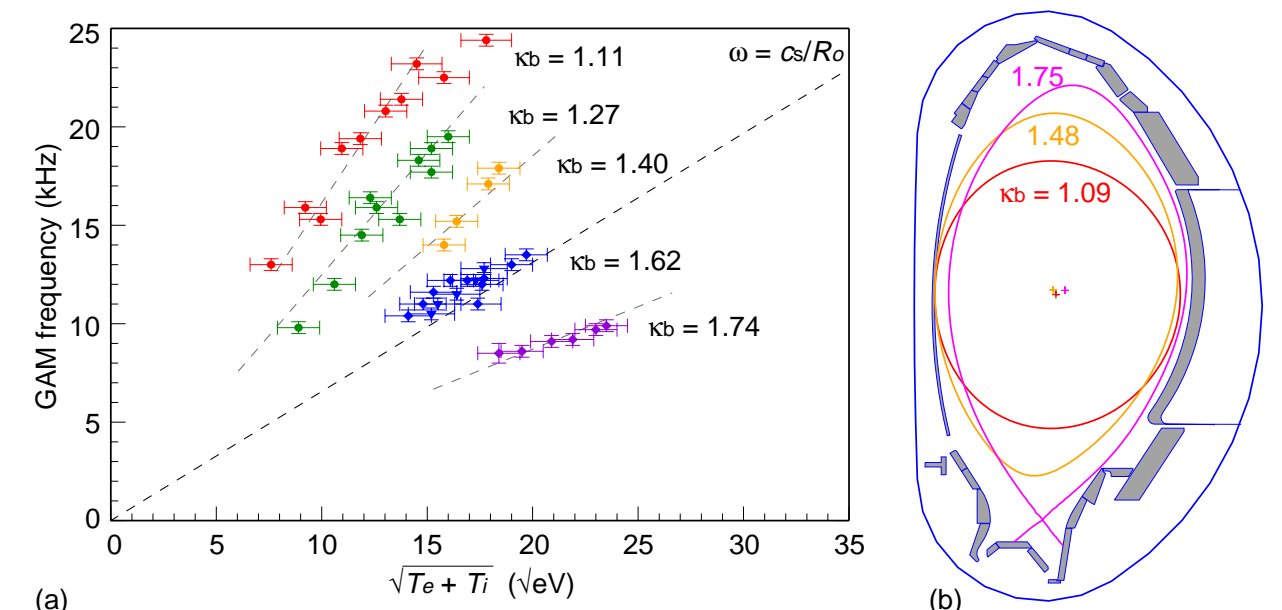

Figure 3. (a) GAM frequency vs $\left(T_{\mathrm{e}}+T_{\mathrm{i}}\right)^{1 / 2}$ for increasing boundary elongation $\kappa_{\mathrm{b}}$ at fixed $q_{95}=3.7$ from edge region $\left(\rho_{\text {pol }}>0.95\right)$ of ohmic $\mathrm{D}_{2}$ plasmas. (b) Selected flux surface boundaries showing extent of $\kappa_{\mathrm{b}}$ variation for limiter and diverted plasmas.

positioned either mid-way above or below the magnetic axis (c.f. figure 1) the diagnostic is usually insensitive to the GAM's $m= \pm 1$ pressure side-band mode structure for standard high elongation $\left(\kappa_{\mathrm{b}}>1.5\right)$ diverted plasmas. However, for low elongation non-diverted configurations, particularly with high magnetic axis, the reflectometer Xmode antenna line-of-sight is closer to the $m=1$ mode maxima, shown in figure 2 by arrows, and a corresponding amplitude $\mathcal{A}$ peak (ie. density fluctuation) is normally seen, figure 2(b), often with harmonics of the mode frequency or other multiple peaks. Such peaks are expected since, while toroidicity couples the $m=0$ flow perturbation with the $m= \pm 1$ side-band, ellipticity and triangularity etc. will couple the $m=0$ to $m= \pm 2$ and higher orders $[23,35]$. Using poloidally distributed reflectometer antennas the structure of the $m=1$ mode was recently investigated in the circular TEXTOR plasmas [18]. However, the precise mode structure of the GAM in complex shaped plasmas is still to be confirmed. In addition, the role of the $\mathrm{X}$-point in the diverted shape is also uncertain, however, it may help to diminish the $m=1$ mode amplitude in the edge by spreading the energy to the higher orders via up-down symmetry breaking.

\section{GAM shape dependence}

Figure $3(\mathrm{a})$ shows the GAM frequency vs $\left(T_{\mathrm{e}}+T_{\mathrm{i}}\right)^{1 / 2}$ for a series of $B_{T}=-2 \mathrm{~T}$, $I_{p} \approx 0.8 \mathrm{MA}$, ohmic Deuterium plasmas with increasing boundary elongation $\kappa_{\mathrm{b}}$ at fixed $q_{95}=3.7$. The data are from radial scans from the edge region, between the pedestal top $\rho_{\text {pol }}>0.95$ and the separatrix/boundary. (The radial coordinate $\rho_{\text {pol }}$ is the squareroot of the normalized poloidal flux.) The electron temperature $T_{\mathrm{e}}$ is measured with electron cyclotron emission (ECE) and Thomson scattering, while the ion temperature $T_{\mathrm{i}}$ is from Li-beam impact excitation spectroscopy. Note Li-beam measurements were not available for all shots, thus $T_{\mathrm{i}}$ is scaled from similar discharges. 

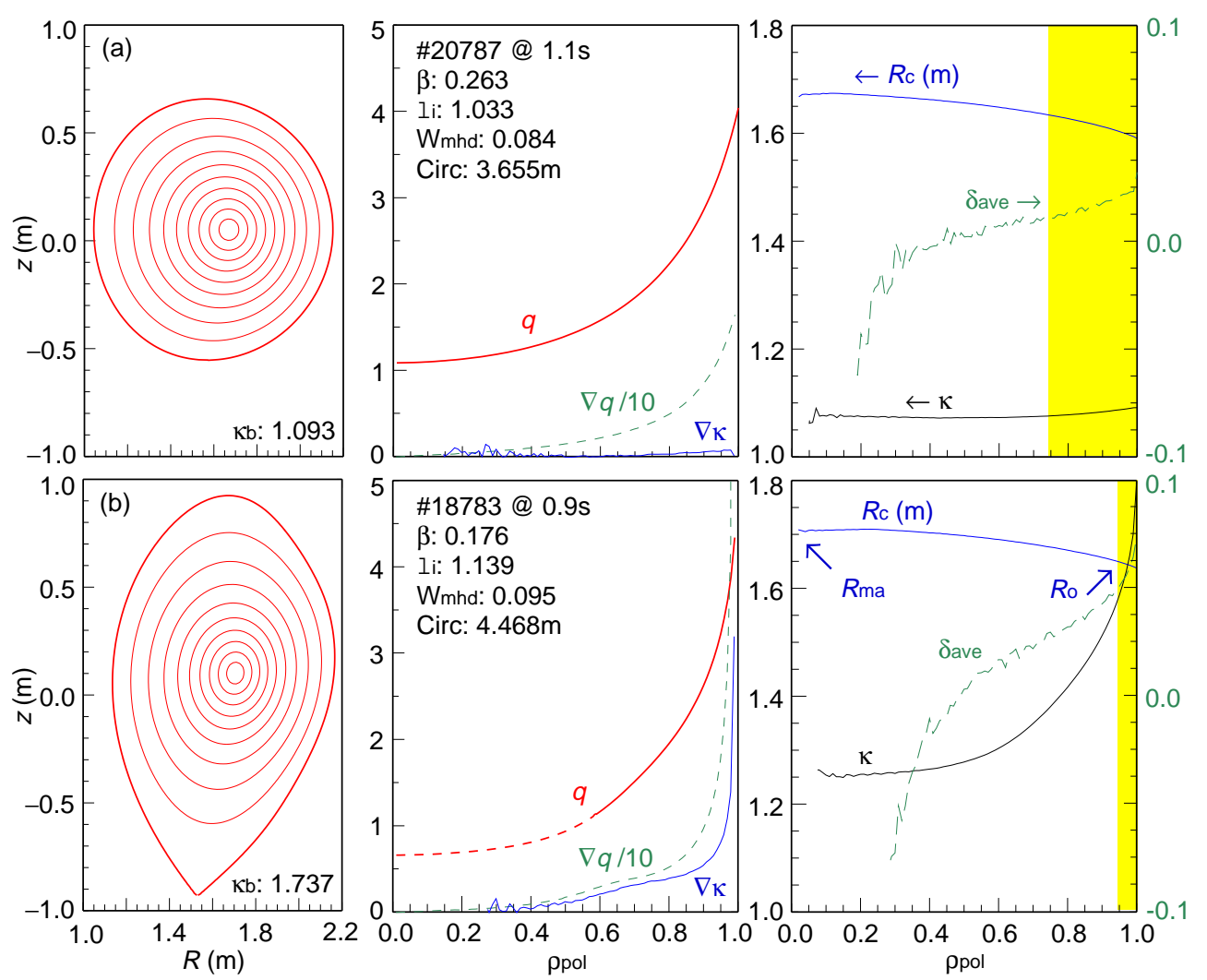

Figure 4. Equilibrium reconstructions from CLISTE code using magnetic data alone with radial profiles of safety factor $q$, flux surface geometric centre radius $R_{\mathrm{c}}$, average triangularity $\delta_{\text {ave }}$ and elongation $\kappa$ with gradients $\nabla q / 10$ and $\nabla \kappa$ for (a) limiter shot \#20787 at $1.1 \mathrm{~s}$ and (b) diverted lower single-null shot \#18783 at $0.9 \mathrm{~s}$, Shaded box indicates GAM extent.

For each shape the GAM frequency scales linearly with the square-root of temperature, that is $\omega_{\mathrm{GAM}}=G c_{\mathrm{s}} / R_{\mathrm{o}}$, where the scale factor $G$ is of the order of unity. The ion sound speed over the geometric major radius $R_{\mathrm{o}}$ scaling has been demonstrated on several devices (c.f. [36]), including the appropriate ion mass variation between hydrogen, deuterium and helium plasmas [18]. However, figure 3(a) also shows a clear inverse dependence on the plasma elongation. (The dashed line is for $G=1$.)

The range of shape variation (elongation $\kappa$ and triangularity $\delta$ ) in AUG is determined by the the active external coils and control system and the internal passive structures [37]. The highest elongation is obtained in a lower single-null divertor configuration, typically between $1.4<\kappa_{\mathrm{b}}<1.75$, as shown in flux boundary poloidal cross-sectional plots in figure $3(\mathrm{~b})$. In non-diverted configurations the elongation can be reduced down to an almost circular cross-section, $1.09<\kappa_{\mathrm{b}}<1.48$ with the plasma touching the inner limiter.

The plasma shape varies with radius. Figure 4 shows magnetic flux surface reconstructions from the CLISTE code [38] using magnetic coil data alone (contours in $\rho_{\text {pol }}$ ) together with radial profiles of the safety factor $q$, the flux surface geometric centre major radius $R_{\mathrm{c}}$, average triangularity $\delta_{\text {ave }}$, elongation $\kappa$ with gradients $\nabla q=d q / d \rho_{\text {pol }}$ 

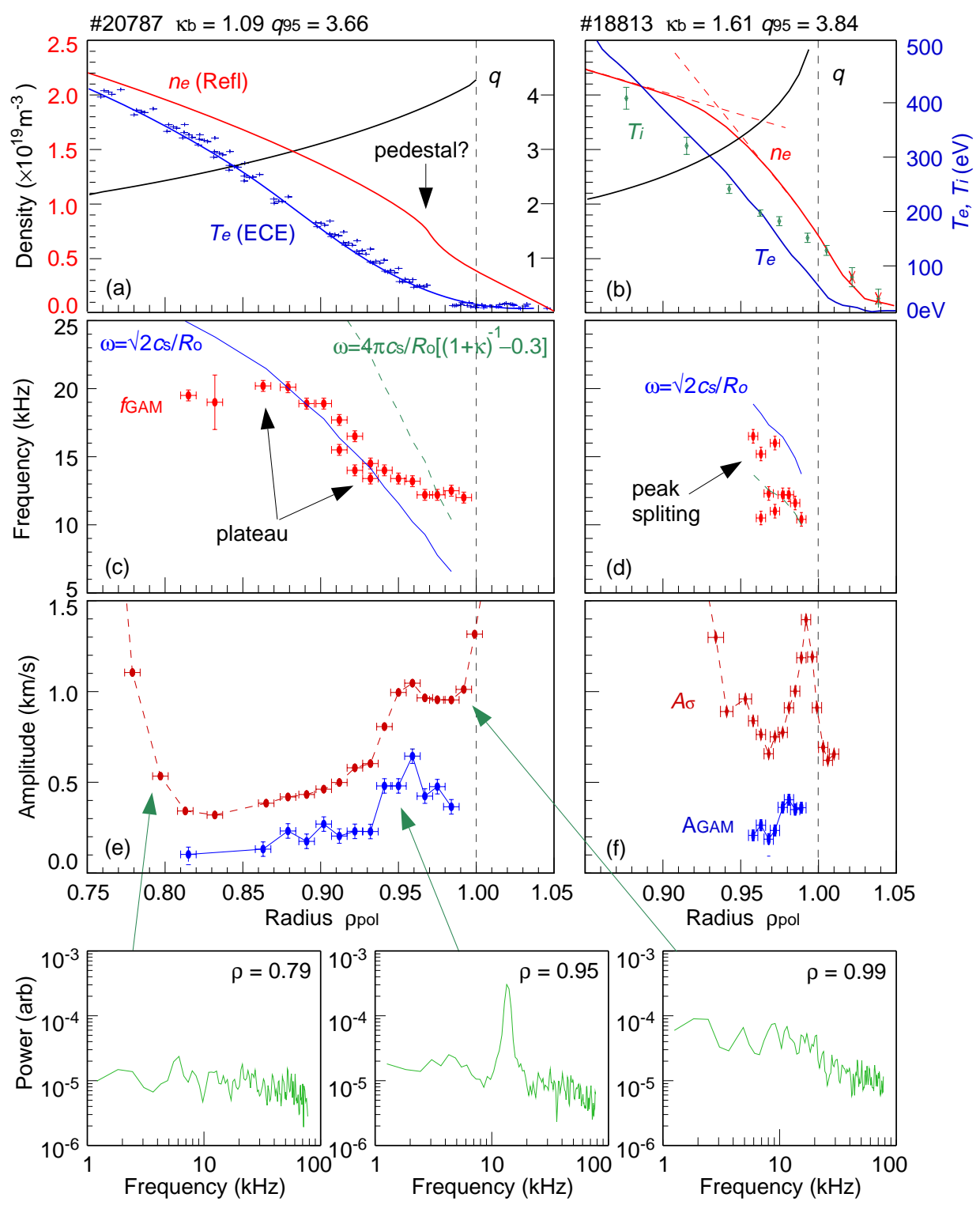

Figure 5. (a \& b) Radial profiles of density $n_{\mathrm{e}}$, temperature $T_{\mathrm{e}}$ and $q$, plus (c \& d) GAM frequency $f_{\text {GAM }}$ and (e \& f) GAM amplitude for ohmic circular limiter shot \#20787 and elongated divertor shot \#18813, with selected $f_{\mathrm{D}}$ spectra for \#20787.

and $\nabla \kappa=d \kappa / d \rho_{\text {pol }}$, for (a) the lowest and (b) highest elongated shapes. Note that $R_{\mathrm{c}}=R_{\mathrm{ma}}$ the magnetic axis for $\rho_{\mathrm{pol}}=0$, and $R_{\mathrm{c}}=R_{\mathrm{o}}$ the boundary geometric axis for $\rho_{\text {pol }}=1$. The radial gradient in both $\kappa$ and $q$ at the plasma edge are stronger the more shaped the plasma.

The remarkable feature of the experimental data in figure 3 is that while the scale factor $G$ clearly varies with the (global) plasma boundary shape, it nevertheless remains independent of the measurement location, despite the dramatic variation in local parameters such as $\kappa, q$ and gradients. This disparity between global and local parameter dependence is a principle topic of this investigation and is addressed in the following sections. 


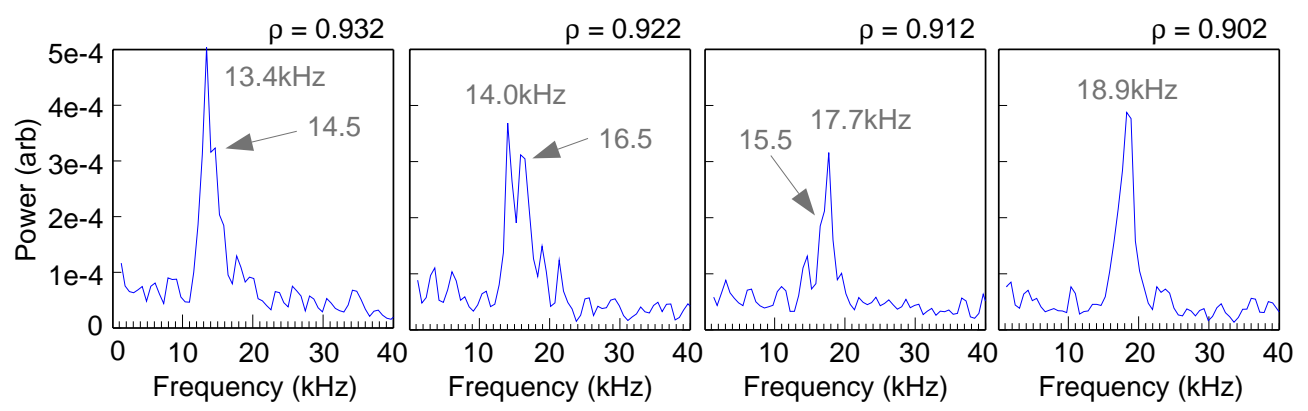

Figure 6. Series of $f_{\mathrm{D}}$ power spectra from decreasing radius showing $f_{\mathrm{GAM}}$ peak splitting at inner zonal edge for ohmic shot \#20787.

\section{GAM radial dependence}

\subsection{Radial zones}

Experimentally the GAM frequency is generally not a smooth monotonic function of radius, unlike temperature (ie. $c_{\mathrm{s}}$ ), or $\kappa$ and $q$, but displays a staircase nature with a series of plateaus a few $\mathrm{cm}$ wide followed by jumps. The plateaus become progressively wider with decreasing radius. Two examples are shown in figure 5 where $f_{\text {GAM }}$ is plotted vs the normalized radius $\rho_{\text {pol }}$ for the circular $\left(\kappa_{\mathrm{b}}=1.09\right)$ limiter ohmic shot \#20787 and the elongated divertor $\left(\kappa_{\mathrm{b}}=1.61\right)$ shot $\# 18813$, together with the corresponding density, temperature and $q$ profiles. Similar frequency plateaus have also been observed in JFT-2M [14] and in numerical simulations [25].

The frequency plateaus generally coincide with regions of enhanced GAM amplitude $A_{\text {GAM }}$ (peak-to-peak velocity perturbation), shown in figure 5 (e \& f), which suggests a series of nested zonal flow layers where the mode phase locks across each zone. When the GAM intensity is weak between zones the mode unlocks and its frequency increases with $c_{\mathrm{s}}$. Also shown in figure 5 is the standard deviation in the Doppler frequency fluctuations $A_{\sigma}$, i.e. the total amplitude of flow perturbations - GAM plus background. Dominant $A_{\mathrm{GAm}}$ peaks are reflected in the standard deviation, but in the core there is a rapid rise in $A_{\sigma}$ which might suggest enhanced random shearing activity [21]. It is also noticeable that the background random fluctuations are also higher in the diverted shaped plasmas.

\subsection{GAM peak splitting}

At the edge of each plateau the GAM intensity drops and the GAM frequency jumps, usually with splitting in the GAM spectral peak. Figure 6 shows a sequence of $f_{\mathrm{D}}$ spectra for decreasing radii for the example shot \#20787 in figure 5(c). As the peak splits the lower frequency branch weakens while the higher frequency peak grows and moves away. This peak splitting is very common. In the elongated discharge in figure 5(d) the frequency separation is more substantial. The lower branch also notably moves down in frequency as well as in amplitude. 


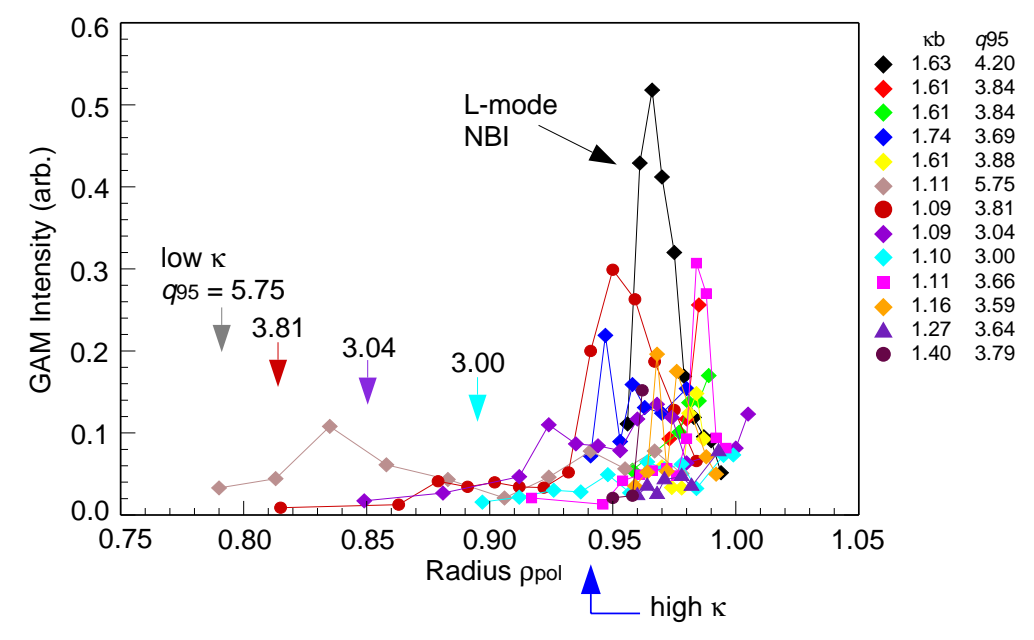

Figure 7. GAM spectral intensity vs radius $\rho_{\text {pol }}$ for a range of ohmic and L-mode shots with various elongation $\kappa_{\mathrm{b}}$ and $q_{95}$.

\subsection{Density pedestal effect}

Figure 5 highlights two other points. In ASDEX Upgrade, the density profile always has a pronounced pedestal around $\rho_{\text {pol }} \approx 0.95$ and $n_{\text {e(ped) }} \sim 2.0-2.5 \times 10^{19} \mathrm{~m}^{-3}$ for divertor configurations. In this configuration GAMs are observed only in the density gradient region and not inside of the pedestal top. The total level of $f_{\mathrm{D}}$ fluctuations $\left(A_{\sigma}\right)$ rises sharply, figure $5(\mathrm{f})$, but there is no coherent activity. Conversely, in the limiter configuration, particularly at low elongation, the density pedestal is weakened and the profile tends towards parabolic. GAMs are now observed well into the plasma, into $\rho_{\text {pol }} \sim 0.75$ - depending on the $q$ profile, (eg. collisionless Landau-like damping). It was previously suggested that the pedestal, or more specifically the second radial derivatives in the profiles, $\partial^{2} / \partial r^{2}$, may act as some form of barrier [11]. It is notable that most shaped devices report GAMs only within a few $\mathrm{cm}$ of the edge, while both the TEXT and TEXTOR circular devices have observed GAMs relatively deep in the plasma $[16,39]$. Unfortunately density profiles were not provided, but parabolic-like profiles are suspected, c.f. [40].

Overlaying the $f_{\text {GAM }}$ profiles in figure $5(\mathrm{c} \& \mathrm{~d})$ is the predicted GAM frequency for a simple large aspect ratio circular plasma, $f_{\mathrm{sc}}=\sqrt{2} c_{\mathrm{s}} /\left(2 \pi R_{\mathrm{o}}\right)$ (solid line) where $c_{\mathrm{s}}$ is defined as before with $\gamma_{\mathrm{i}}=1$, plus a best-fit scaling to the edge GAM database (dashed line) - the edge and core frequency models are discussed in more detail later. Here, the core is defined as the region inside the density pedestal top radius and the edge outside. For the circular plasma the frequency plateaus and jumps are more pronounced inside the pedestal top radius, nevertheless, the mean GAM frequency tends to follow the predicted circular scaling quite closely. However, outside the pedestal top $f_{\mathrm{GAM}}$ diverges from the core scaling $f_{\mathrm{sc}}$, even for the circular plasma, and can be either higher or lower than the simple predicted scaling depending on the plasma shape. 


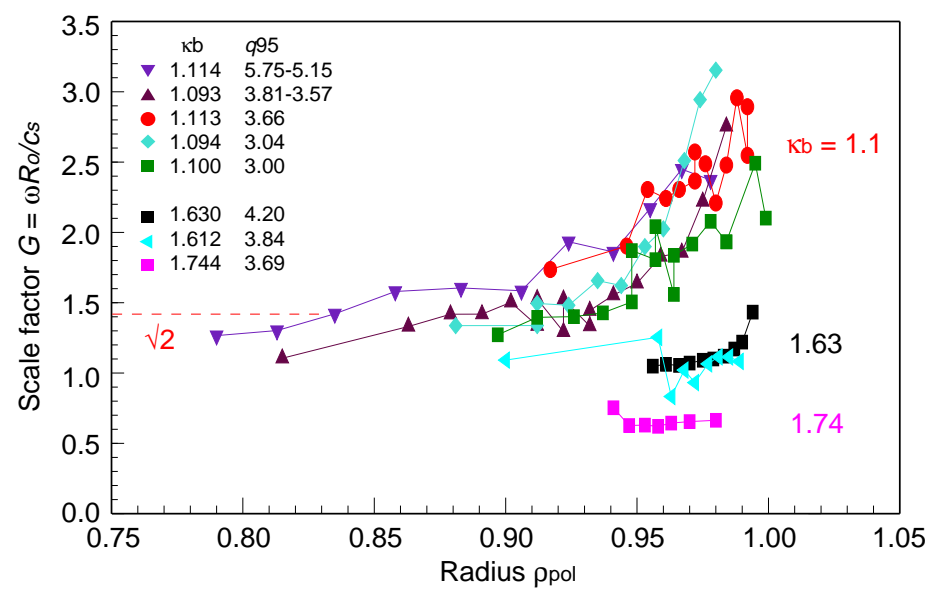

Figure 8. GAM scale factor $G=\omega R_{\mathrm{o}} / c_{\mathrm{s}}$ vs normalized radius $\rho_{\text {pol }}$ for a series of low $\kappa_{b} \sim 1.1$ circular ohmic plasmas with $3.0<q_{95}<5.7$. Plus, selected profiles at $\kappa_{\mathrm{b}}=1.63$ and 1.74

\subsection{GAM localization}

Figure 7 shows a collection of profiles of the GAM spectral peak intensity vs normalized radius $\rho_{\text {pol }}$ for a range of ohmic and L-mode (ECRH and NBI) discharges with various elongation $1.09<\kappa_{\mathrm{b}}<1.74$ and edge safety factors $3.0<q_{95}<4.2$. Each profile displays one or more radial peaks, the most intense being nearly always in the edge density gradient region. Only for the ohmic low $\kappa_{\mathrm{b}}$, high $q_{95}$ case is the GAM stronger in the core region. Indeed, the GAM intensity and radial extent increases with $q_{95}$ consistent with Landau damping proportional to $\exp \left(-q^{2}\right)[41,22,35]$. For divertor Xpoint discharges, i.e. high $\kappa_{\mathrm{b}}>1.4$, no GAM peaks are observed beyond approximately $\rho_{\text {pol }}<0.94$ - marked by the arrow in figure 7 .

There is no clear preferred radial position for the GAM maxima, and there is no alignment with any rational $q$ surface. The radial peaks tend to become broader towards the core. The effect of increased turbulence drive with additional heating is also evident in the stronger L-mode peaks. The parameter dependence of the GAM amplitude is discussed more fully in a separate paper.

\section{Core GAM behaviour}

For inner limiter configurations the edge density pedestal in AUG becomes weaker (to almost non-existent) at $n_{\mathrm{e} \text { (ped) }} \sim 1 \times 10^{19}$ compared to $>2.0 \times 10^{19} \mathrm{~m}^{-3}$ for the divertor configuration. The result is that GAMs are now observed further into the core, as far as $\rho_{\text {pol }} \sim 0.75$ at high $q_{95}$, as shown in figure 8 where the scale factor $G=\omega_{\mathrm{GAM}} R_{\mathrm{o}} / c_{\mathrm{s}}$ (ie. normalized GAM frequency) is plotted against $\rho_{\text {pol }}$ for a series of low $\kappa_{\mathrm{b}} \sim 1.1$ ohmic deuterium shots with $q_{95}$ ranging between 3.0 and 5.7 (obtained by varying the plasma current). In the edge there is some scatter in $G$ due to the $f_{\text {GAM }}$ plateaus and jumps, and $q$ variation, but the profiles become smoother with decreasing radius and tend towards 


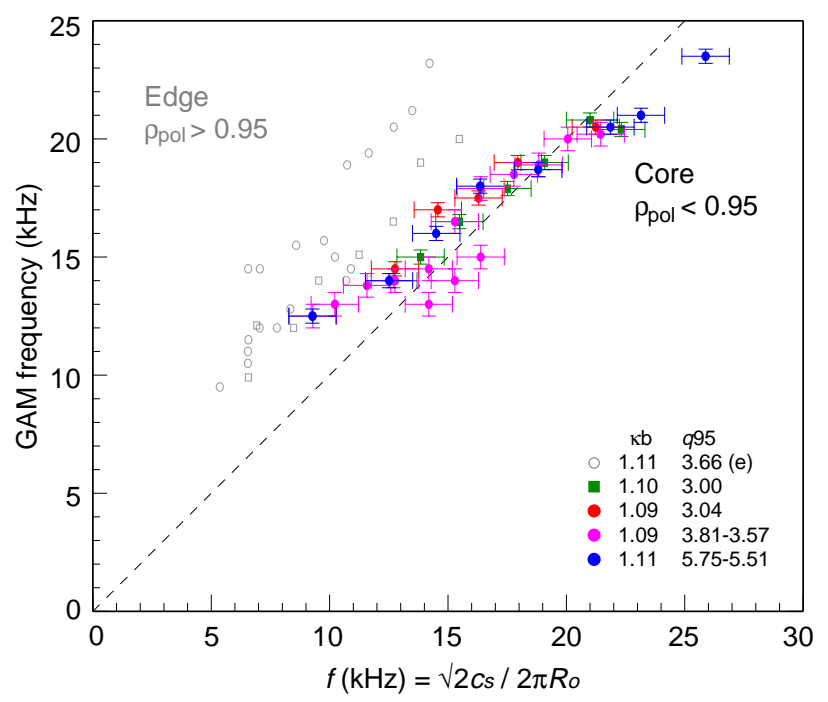

Figure 9. Core $f_{\mathrm{GAM}}$ vs $f=\sqrt{2} c_{\mathrm{s}} /\left(2 \pi R_{\mathrm{o}}\right)$.

a constant $G$ value of around $\sqrt{2}$. Also shown are comparative profiles with $\kappa_{\mathrm{b}}=1.63$ and 1.74, where $G$ starts at a lower value in the edge but are suggestive in appearing to rise towards the same asymptotic $G$ value around the top of the pedestal, and in one rare case inside the pedestal. Unfortunately the current database of core measurements is rather limited to a range of low $\kappa$ cases, nevertheless, there does not appear, within experimental uncertainty, to be a discernable variation in the core asymptotic $G$ value with either $\kappa$ or $q$ (c.f. also figure 11 below).

In figure 9 the GAM frequency is plotted against the simple circular scaling $f_{\text {sc }}$ for the core GAM database with $\kappa_{b}<1.16$ and all $q_{95}$ values. The agreement for GAM peaks inside $\rho_{\text {pol }} \approx 0.95$ is rather good. At the lower temperatures, i.e. towards the edge, the points tend to move away from the perfect scaling, and for GAM peaks outside $\rho_{\text {pol }} \approx 0.95$ (marked in open grey symbols) they do not fit the circular scaling at all but follow a separate edge frequency scaling. This indicates that, even for $\kappa_{b} \rightarrow 1$, the physics is different in the density gradient region.

\section{Edge GAM behaviour}

\subsection{Local parameter dependence}

Plotting the edge GAM data of figure 3 in terms of the scale factor $G$ against normalized radius $\rho_{\text {pol }}$ in figure 10 for various elongation at fixed $q_{95} \approx 3.7$ shows more clearly the constant nature of $G$ (aside from the frequency plateaus) across the edge gradient region, for all plasma elongation, except the circular shape $\kappa \sim 1.1$ at the very edge. This is intriguing since it implies that any local shape and $q$ effects are either mostly balanced, or they are irrelevant.

Theory and simulation studies suggest several geometric parameters of potential relevance: ellipticity $\kappa$, the safety factor $q$, triangularity $\delta$, the inverse aspect ratio 


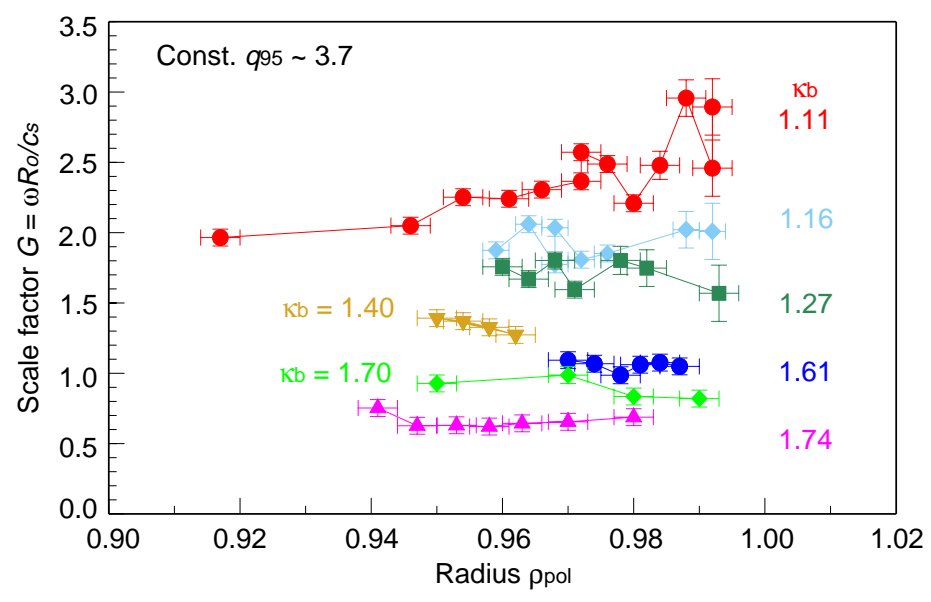

Figure 10. GAM scale factor $G=\omega R_{\mathrm{o}} / c_{\mathrm{S}}$ vs normalized radius $\rho_{\text {pol }}$ for various ohmic $\mathrm{D}_{2}$ plasma elongation $\kappa_{b}$ at fixed $q_{95} \approx 3.7$.
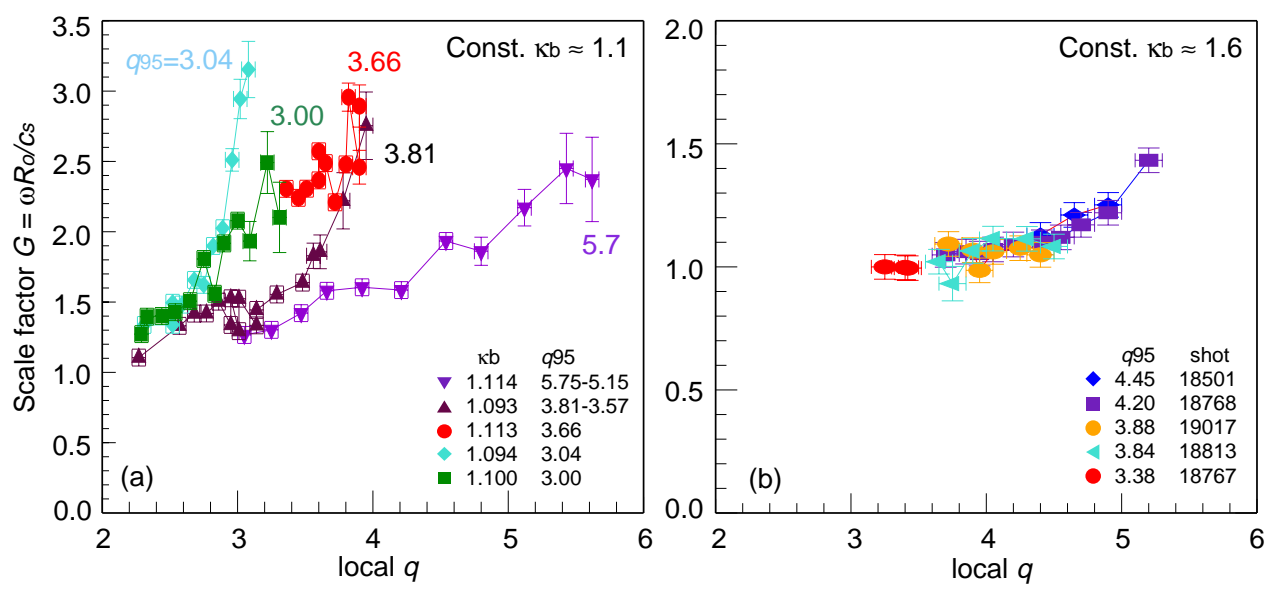

Figure 11. GAM scale factor $G=\omega R_{\mathrm{o}} / c_{\mathrm{s}}$ vs local $q$ for (a) $\kappa_{\mathrm{b}}=1.1$ and (b) $\kappa_{\mathrm{b}}=1.6$.

$\epsilon=r / R$, flux surface displacement (Shafranov) $\Delta=R_{\mathrm{o}}-R_{\mathrm{c}}$, and their gradients $d \kappa / d r, d \Delta / d r$ and via the turbulence possibly $d q / d r$ etc.

In figure 11 the scale factor $G$ is plotted against the local $q$ value for two groups of edge GAMs: (a) low $\kappa_{\mathrm{b}}=1.1$ and (b) high $\kappa_{\mathrm{b}}=1.6$ with various $q_{95}$. At high shaping there is only a very weak direct variation with $q$, but as $\kappa$ is reduced the $q$ variation becomes stronger. At low $q$, ie. core, the sensitivity again disappears and $G$ tends to constant. The spread in the curves with $q_{95}$ at low $\kappa$ suggests that $q$ alone is not the relevant parameter. Replotting $G$ against normalized $q / q_{95}$ in figure 12 (a) however, reduces the low $\kappa$ data to roughly a single curve. In fact there are a set of nested curves with increasing $\kappa_{\mathrm{b}}$. The $q / q 95$ dependence might suggest that the $q$ profile shape, i.e. the magnetic shear $s=d q / d r$ or $d \kappa / d r$ maybe important. Or put in more general terms there is a radial dependence in the curvature operator. However, testing for $s$ or normalized shear $\hat{s}=(r / q) d q / d r$ did not reveal any clear dependence. Likewise no systematic dependence on the triangularity or $\Delta$ was found.

Figure 12(b) shows $G$ vs local $\kappa$ at fixed $q_{95}=3.7$ for a range of ohmic shots 

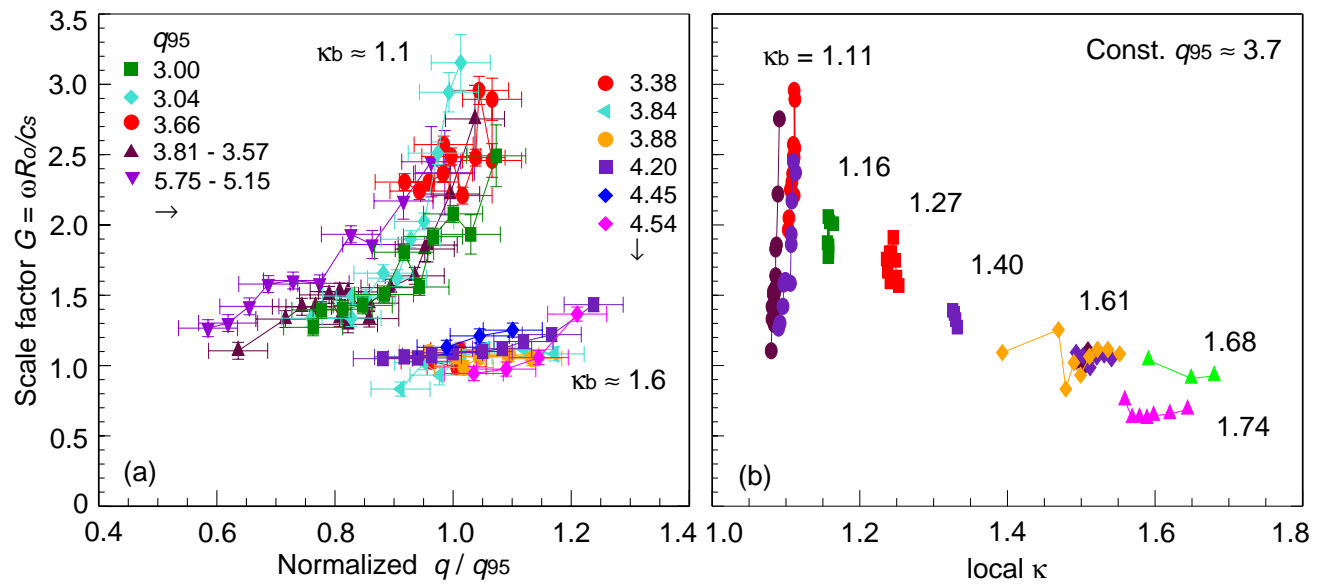

Figure 12. GAM scale factor $G=\omega R_{\mathrm{o}} / c_{\mathrm{s}}$ vs (a) normalized $q / q_{95}$ for various $q_{95}$ for low $\kappa_{\mathrm{b}}=1.1$ and high $\kappa_{\mathrm{b}}=1.6$, and (b) local elongation $\kappa$ with $q_{95} \approx 3.7$ for various plasma boundary elongation.
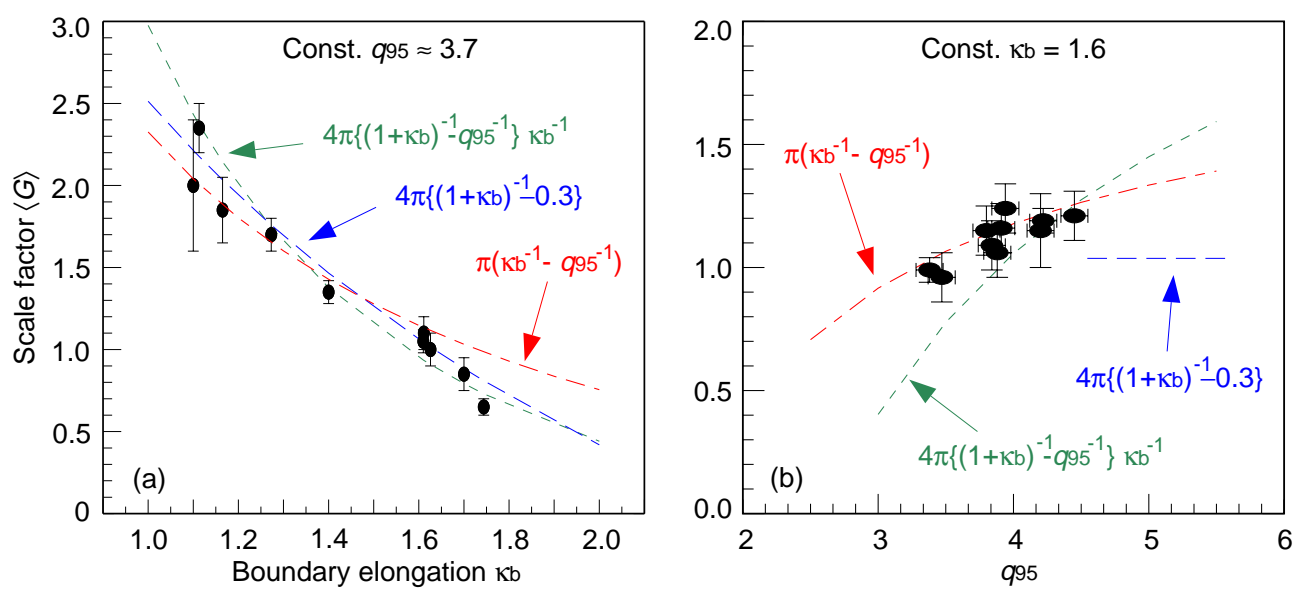

Figure 13. Radially averaged edge GAM scale factor $\langle G\rangle$ vs (a) boundary elongation $\kappa_{\mathrm{b}}$ for constant $q_{95} \approx 3.7$ and (b) $q_{95}$ for constant $\kappa_{b} \sim 1.6$.

with increasing boundary elongation $1.11<\kappa_{\mathrm{b}}<1.74$. As expected, $G$ generally decreases with increasing $\kappa_{\mathrm{b}}$, but within a radial sweep the $G$ variation changes from vertical (sensitive) to horizontal (insensitive) with increasing $\kappa_{\mathrm{b}}$ - as might be expected from the $\kappa$ and $q$ radial profiles in figure 4 . At low $\kappa_{\mathrm{b}}, \kappa(r)$ is almost constant while $q(r)$ is changing, but at high $\kappa_{\mathrm{b}}$, both $\kappa(r)$ and $q(r)$ vary. This transition from $q$ sensitive to insensitive with increasing $\kappa$ might suggest a frequency scaling of the form: $G \propto(1 / \kappa-c / q)$ with appropriate constants $c$.

\subsection{Global parameter dependence}

The alternative interpretation of the data in figure 12 is that, for the restricted radial zonal range in the edge, some mean or representative value of $\kappa$ and $q$, together perhaps with gradients maybe sufficient or more appropriate to parameterize the GAM frequency. 


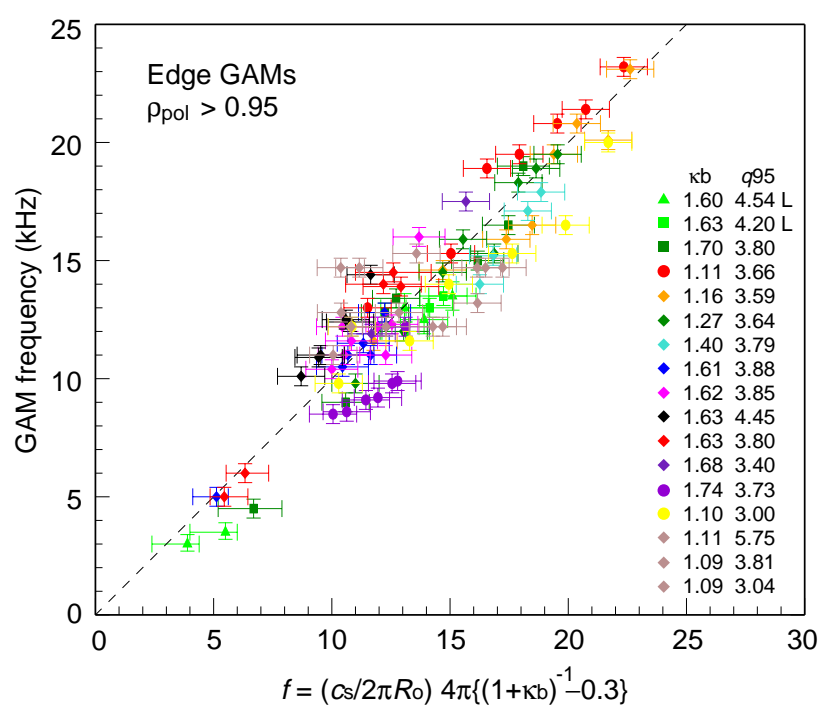

Figure 14. Measured $f_{\mathrm{GAM}}$ vs model frequency $f=\left(c_{\mathrm{s}} / 2 \pi R_{\mathrm{o}}\right) 4 \pi\left(\left[1+\kappa_{b}\right]^{-1}-\epsilon_{o}\right)$ for edge GAM database $\rho_{\text {pol }}>0.95$ with $\epsilon_{o}=0.3$.

In figure $13(\mathrm{a})$ the radially averaged $\langle G\rangle$ is plotted vs the boundary elongation $\kappa_{\mathrm{b}}$ showing again a clear inverse dependence. Conversely, holding $\kappa_{\mathrm{b}} \approx 1.6$ constant and varying the $q$ profile reveals a weaker direct dependence on $q_{95}$, as shown in figure 13(b). Unfortunately the range of variation in $q_{95}$ is rather limited so it is difficult to discriminate between potential models. As shown in figure 12 the sensitivity to $q$ becomes stronger at lower elongation.

A variety of reciprocal and linear fits to the data are possible. Overlayed in figure 13 are three potential models. The (red) dash-dot line is for $G=\pi\left(\kappa_{\mathrm{b}}^{-1}-q_{95}^{-1}\right)$. This gives a reasonable fit to the lower end of the elongation scan and the best fit to the $q$ scan. The other two models assume a $(1+\kappa)^{-1}$ variation. This might appear if the mode wavelength were to scale with the poloidal circumference - although there is no strong theoretical background for this. The (green) dotted line retains a $q_{95}$ term, however, now an additional $\kappa_{\mathrm{b}}^{-1}$ term is necessary: $G=4 \pi\left[\left(1+\kappa_{\mathrm{b}}\right)^{-1}-q_{95}^{-1}\right] \kappa_{\mathrm{b}}^{-1}$. This gives the best fit to the elongation scan, but a rather poor fit to the $q$ scan. It should be noted that the negative $q^{-1}$ dependence, although not obvious in simple fluid theory, can arise from the shear or other geometry factors, while the second $\kappa^{-1}$ factor might contain a triangularity dependence - since $\delta$ tends to increase with elongation. The most interesting fit is the (blue) dashed line which is without a $q_{95}$ term: $G=4 \pi\left[\left(1+\kappa_{\mathrm{b}}\right)^{-1}-\epsilon_{o}\right]$. Here $\epsilon_{o}=0.3$ is a constant term which is very close to the average inverse aspect ratio $\epsilon=r / R_{\mathrm{c}} \sim a / R_{o} \sim \mathcal{O}(0.3)$ over the edge region of AUG plasmas. It gives a reasonable fit to the $\kappa$ scan and is within the error bars of the $q$ scan. 


\begin{tabular}{|l|l|l|}
\hline & Model & RMSE \\
\hline \multirow{2}{*}{$\begin{array}{l}\text { Edge } \\
\rho_{\text {pol }}>0.95 \\
\text { All } \kappa_{\mathrm{b}}\end{array}$} & $\omega=4 \pi\left[\left(1+\kappa_{\mathrm{b}}\right)^{-1}-\epsilon_{\mathrm{o}}\right] c_{\mathrm{s}} / R_{\mathrm{o}}$ & 1.715 \\
& $\omega=\left(4 \pi / \kappa_{\mathrm{b}}\right)\left[\left(1+\kappa_{\mathrm{b}}\right)^{-1}-q_{95}^{-1}\right] c_{\mathrm{s}} / R_{\mathrm{o}}$ & 2.393 \\
\cline { 2 - 3 } & $\left.\omega=\pi\left(\kappa_{\mathrm{b}}^{-1}-q_{95}^{-1}\right) c_{\mathrm{s}} / R_{\mathrm{o}}-1\right) c_{\mathrm{s}} / R_{\mathrm{o}}$ & 2.504 \\
\hline \multirow{2}{*}{$\begin{array}{l}\text { Core } \\
\rho_{\text {pol }}<0.95 \\
\kappa_{\mathrm{b}}<1.16\end{array}$} & $\omega=\left(2+q^{-2}\right)^{1 / 2} c_{\mathrm{s}} / R_{\mathrm{o}}$ & 2.960 \\
\cline { 2 - 4 } & $\omega=\sqrt{2} c_{\mathrm{s}} / R_{\mathrm{o}}$ & 4.504 \\
\hline
\end{tabular}

Table 1. Root mean square error RMSE between measured GAM frequency and predicted frequency from various models for the edge $\rho_{\text {pol }}>0.95$ and core $\rho_{\text {pol }}<0.95$ GAM databases.

\section{GAM frequency scaling}

Various heuristic models for the GAM frequency, including those presented above, were tested against the full database of ohmic and L-mode GAM frequencies. Figure 14 shows the measured $f_{\mathrm{GAM}}$ against the best model frequency $f=\left(c_{\mathrm{s}} / 2 \pi R_{\mathrm{o}}\right) 4 \pi\left[\left(1+\kappa_{\mathrm{b}}\right)^{-1}-\epsilon_{o}\right]$ for edge GAM peaks outside $\rho_{\text {pol }}>0.95$. The agreement is quite good with a root mean square error $\left(\mathrm{RMSE}=\left\langle\left(f_{\mathrm{GAM}}-f\right)^{2}\right\rangle^{1 / 2}\right)$ of 1.715 , which is of the same order as the typical error bar $\sigma \approx 1.5$ in the model frequency. Here, the largest source of uncertainty is in the measured temperatures $T_{\mathrm{e}}$ and $T_{\mathrm{i}}$ used to compute the sound speed.

Table 1 summarizes the fit to a selection of the best models for both the edge and core GAM databases. For the edge fits the sample population is $N=113$ while for the core fits $N=26$. Extreme outliers were removed but otherwise the sample includes all systematic tends due to frequency plateaus and steps. For the edge region the best fit is to the simplest model with no explicit $q$ term, but a fixed correction term. The fit progressively degrades with the inclusion of more terms, and notably the fits worsen with use of local rather than global parameters. The improvement of the fit without $q$ implies that, at best it plays only a weak role, or that it introduces other dependencies which are not well described by the $q^{-1}$ form. However, other formulations, including $q / q_{95}$, produced worse fits. The poor $q$ sensitivity may seem contrary to the large $G$ variation in figure $12(\mathrm{a})$, however, since $G$ is computed from a ratio with the main 
uncertainty in the denominator, this translates to larger variations in $G$ as it increases with decreasing $\kappa_{\mathrm{b}}$, while the RMSE as an expression of the frequency difference is a more direct indicator of the model agreement.

For the core database a series of variations on the basic model were tested. The best fit with an RMSE of 1.615 was for the simplest circular model. Including the parallel $q$ term marginally degraded the RMSE to 1.702 (or 1.619 using $q_{95}$ ). Replacing $R_{\mathrm{o}}$ with $R_{\mathrm{c}}$ moves the data points in figure 9 slightly to the left, which improves the core agreement by $\sim 0.1 \mathrm{kHz}$, but also degrades the fit towards the pedestal top. For the edge gradient region it has a negligible effect. Overall, using $R_{\mathrm{c}}$ raises the core RMSE marginally to 1.623. For all the results presented $c_{\mathrm{s}}$ was calculated with $\gamma_{\mathrm{i}}=1$. Setting $\gamma_{\mathrm{i}}=7 / 4$ raises the simple circular RMSE from 1.615 to 3.161 for the core points. However, if the edge database is restricted to $\kappa_{\mathrm{b}}<1.16$ then the simple circular model gives an RMSE of $3.641(N=40)$ for $\gamma_{\mathrm{i}}=7 / 4$ compared to 5.258 for $\gamma_{\mathrm{i}}=1$.

For both the core and edge databases no explicit dependence on either triangularity or magnetic shear could be determined within the measurement error. Likewise no clear density and/or temperature scale length dependence was found.

As a crosscheck the core and edge databases were tested against their corresponding best counterpart model. The resulting RMSEs of 8.710 and 5.294 respectively confirm that the simple models so far considered are not universal and are limited to their respective regimes.

\section{Summary and discussion}

The observation of GAMs is ubiquitous in ohmic and L-mode AUG discharges. Providing there is a sufficient level of turbulence (to both drive the GAM and to provide a measurable backscatter signal for the Doppler reflectometer diagnostic), and the plasma collisionality is not too high (i.e. $\omega_{\mathrm{GAM}}>\nu_{\mathrm{ii}}$ ), then a GAM will be found in some region of the tokamak. It is nearly always strongest in the edge density gradient region towards the top of the pedestal. In diverted AUG plasmas there is always a distinct density pedestal, which appears to restrict the inner radial extent of the GAM. However, for non-diverted plasmas of almost circular shape touching the inner limiter, the density pedestal is strongly reduced and the GAM is seen to extend towards the plasma midradius. Its extent now seemingly limited only by collisionless Landau damping prescribed by the $q$ profile.

Radially the GAM frequency $\omega_{\text {GAM }}$ profile shows a series of steps or plateaus which become wider with decreasing radius, or profile gradient. Around each plateau edge or step the GAM frequency spectrum splits into two branches, with the higher frequency peak growing while the lower one diminishes. The plateaus are also accompanied by peaks in the GAM intensity, which together with the frequency plateaus suggest zonal rings. Nevertheless, the mean or underlying GAM frequency scales with $c_{\mathrm{s}} / R_{\mathrm{o}}$.

For circular plasmas the GAM frequency in the core region scales close to the theoretical prediction of $\omega \approx \sqrt{2} c_{\mathrm{S}} / R_{\mathrm{o}}$. Including the $1 / q^{2}$ term due to parallel coupling 
marginally degrades the overall agreement (mostly for the higher temperature / deeper core points), but since $q>1$ this term only plays a marginal role. However, towards the edge of the circular plasmas the GAM frequency radically diverges from the predicted scaling with frequencies significantly higher than expected.

Towards the edge the plasma collisionality increases, and in gradient region of diverted plasmas $T_{\mathrm{i}}>T_{\mathrm{e}}$ by $20 \%$ or more [42] while in the core $T_{\mathrm{i}} \approx T_{\mathrm{e}}$. Further, the good fit for the circular core GAM frequencies was obtained with a specific heat ratio of $\gamma_{\mathrm{i}}=1$ (isothermal) while a $\gamma_{\mathrm{i}}=7 / 4$ (dissipation free adiabatic) produced a much worse fit. This implies that the ion temperature fluctuation contributions to $\gamma=1\left[\tilde{n}_{i}\right]+\frac{1}{2}\left[\tilde{T}_{i||}\right]+\frac{1}{4}\left[\tilde{T}_{i \perp}\right]$ (where the respective sources of the terms are indicated in square brackets) are suppressed due to the parallel dissipation at the low $q$ leading to dominant collisionless Landau damping [41]. In the edge a $\gamma_{\mathrm{i}}=7 / 4$ improves the fit (in particular the scale factor $G$ variation with $q$ is substantially flattened), however it is still not sufficient to fully reconcile the core and edge GAM frequency behaviour under one model.

Raising the elongation now decreases the edge GAM frequency as $f_{\mathrm{GAM}} \propto(1+\kappa)^{-1}$. The previous variation with $q$ all but disappears and the scale factor $G$ is constant in radius. Unfortunately, there are insufficient core measurements for elongated plasmas so it is not possible to determine if the same $\kappa$ dependence applies, but the indications so far are that it does not. Nevertheless, a simple heuristic formula which acceptably fits the edge GAM frequency behaviour has been deduced requiring only two global parameters: the reciprocal $\left(1+\kappa_{\mathrm{b}}\right)$ and a constant $\epsilon_{o}$. Linear theory $[22,28]$ suggests a $\kappa^{-1}$ dependence rather than the experimentally observed $\left(1+\kappa_{\mathrm{b}}\right)^{-1}$. This suggests either the theory is deficient, or that the $\left(1+\kappa_{\mathrm{b}}\right)$ term incorporates the effects of higher order factors such as $\nabla \kappa$. The constant $\epsilon_{o}$ is very close to the average inverse aspect ratio for the GAM peak radius $r / R_{\mathrm{c}} \approx 0.3$. However, to test this hypothesis requires a wider range of measurements from different aspect ratio machines. Additional experimental input may also help to determine the most appropriate value for $\gamma_{\mathrm{i}}$ in the edge. From numerical simulations there are indications that a $\kappa>1$ and/or large $r(d \kappa / d r)$ tend to enhance the effects of parallel dynamics and magnetic shear [30], i.e. an increased adiabatic coupling. The absence of a $q$ dependence in the edge fit is also consistent with a strong collisionless Landau damping [41]. Either way, adiabatic corrections may be incorporated in the heuristic formula with $c_{\mathrm{s}} \rightarrow \gamma c_{\mathrm{s}}$ via an effective $\gamma=\sqrt{\frac{1+\gamma_{\mathrm{i}} \tau}{1+\tau}}$ where $\tau=T_{\mathrm{i}} / T_{\mathrm{e}}$.

The elongation and triangular shaping of the plasma are also expected to lead to coupling of the $m=0$ flow to additional $m= \pm 2, \pm 3$.. pressure modes, and indeed spectral peaks and harmonics are observed in the density fluctuations in these situation.

Between the limiter and divertor shape the density pedestal top appears to act as a boundary, yet no clear profile scale length $\left(R / L_{\mathrm{T}}\right.$ etc.) or $\nabla n$ dependence on the frequency is currently evident in either experimental data or theory. Nevertheless, the edge region appears to behave differently from the core - although connected with a 
smooth transition - and can only be related to local effects such as the higher turbulence drive (perhaps pushing the GAM frequency), higher collisionality, larger $q$ and magnetic shear, larger vorticity and the stronger $E_{r}$ shear present, not to mention the coupling effects to Alfvén and sound waves etc. [8, 28]. It should also be noted that the presence of an X-point in the diverted shape will destroy the up-down symmetry leading to stronger Stringer spin-up effects [43] which may affect the frequency.

Further theory and numerical simulation in realistic shaped equilibria are necessary to clarify the GAM behaviour in the plasma edge. In particular, a tested (analytic) expression for $f_{\mathrm{GAM}}$ in shaped diverted plasmas using readily measurable parameters is required if one wants, as recently proposed, to use GAM spectroscopy to determine the ion mass [44] in real tokamak edges where the GAMs are actually observed.

\section{Acknowledgments}

We thank J.Schirmer, W.Suttrop and E.Schmid for assistance with reflectometer hardware; B.Kurzan for validation of TS density profiles; C.Maggi for CXRS data; M.Reich for Li-beam $T_{\mathrm{i}}$ measurements, and P.Angelino, A.Bottino and F.Jenko for fruitful discussions.

\section{References}

[1] Lin Z, Hahm T S, Lee W W, Tang W M and White R B 1998 Science 2811835

[2] Diamond P H and Kim Y-B 1991 Phys. Fluids B 31626

[3] Ramisch M, Stroth U, Niedner S and Scott B 2003 New J. Phys. 512

[4] Scott B 2003 Phys. Lett. A $\mathbf{3 2 0} 53$

[5] Hahm T S, Beer M A, Lin Z, Hammett G W, Lee W W and Tang W M 1999 Phys. Plasmas 6922

[6] Diamond P H, Itoh S-I, Itoh K and Hahm T S 2005 Plasma Phys. Control. Fusion 47 R35

[7] Hahm T S 2002 Plasma Phys. Control. Fusion 44 A87

[8] Scott B D 2005 New J. Phys. 792

[9] Winsor N, Johnson J L and Dawson J M 1968 Phys. Fluids 112448

[10] Grimm R C and Johnson J L 1975 J. Computational Phys. 17192

[11] Conway G D et al 2005 Plasma Phys. Control. Fusion 471165

[12] McKee G R, Gupta D K, Fonck R J, Schlossberg D J, Shafer M W, and Gohil P 2006 Plasma Phys. Control. Fusion 48 S123

[13] Yan L W et al 2007 Nucl. Fusion 471673

[14] Ido T et al 2006 Plasma Phys. Control. Fusion 48 S41

[15] Nagashima Y et al 2006 Plasma Phys. Control. Fusion 48 S1

[16] Schoch P M, Connor K A, Demers D R and Zhang X 2003 Rev. Sci. Instrum. 74 1846. Also Schoch P M, Connor K A and Demers D R 2001 Bull. Am. Phys. Soc. 468

[17] Vershkov V A et al 2005 Nucl. Fusion 45 S203

[18] Krämer-Flecken A, Soldatov S, Koslowski H R, Zimmermann O and TEXTOR Team 2006 Phys. Rev. Lett. 97045006

[19] Xu G S, Wan B N and Song M 2002 Phys. Plasmas 9150

[20] Hahm T S, Burrell K H, Lin Z, Nazikian R and Synakowski E J 2000 Plasma Phys. Control. Fusion 42 A205

[21] Kim E-J and Diamond P H 2004 Phys. Plasmas 11 L77

[22] Watari T, Hamada Y, Fujisawa A, Toi K and Itoh K 2005 Phys. Plasmas 12062304 
[23] Hallatschek K 2006 Proc. 21st IAEA Fusion Energy Conf. (Chengdu) (Vienna: IAEA) IAEA-CN149/TH/2-6, http://www-pub.iaea.org/MTCD/Meetings/FEC2006/th_2-6.pdf

[24] Hallatschek K and Biskamp D 2001 Phys. Rev. Lett. 861223

[25] Miyato N, Kishimoto Y and Li J 2004 Phys. Plasmas 115557

[26] Itoh K, Hallatschek K and Itoh S-I 2005 Plasma Phys. Control. Fusion 47451

[27] Novakovskii S V, Liu C S, Sagdeev R Z and Rosenbluth M N 1997 Phys. Plasmas 44272

[28] Hallatschek K 2007 Plasma Phys. Control. Fusion 49 B137

[29] Angelino P et al 2006 Proc. 33rd EPS Conf. Plasma Phys. (Rome), ECA 30I, P1.195

[30] Kendl A and Scott B D 2006 Phys. Plasmas 13012504

[31] Conway G D et al 2006 Proc. 21st IAEA Fusion Energy Conf. (Chengdu) (Vienna: IAEA) IAEACN-149/EX/2-1, http://www-pub.iaea.org/MTCD/Meetings/FEC2006/ex_2-1.pdf

[32] Conway G D, Tröster C, Scott B, Hallatschek K and ASDEX Upgrade Team 2007 Proc. 34th EPS Conf. Plasma Phys. (Warsaw) ECA vol 31F (Mulhouse: EPS) O-4.009

[33] Conway G D, Schirmer J, Klenge S, Suttrop W, Holzhauer E and ASDEX Upgrade Team 2004 Plasma Phys. Control. Fusion 46951

[34] Conway G D et al 2007 Proc. 8th Intl. Reflectometry Wksh. for fusion plasma diagnostics IRW8 (St.Petersburg) p30, http://www.aug.ipp.mpg.de/IRW/IRW8

[35] Angelino P et al 2006 Plasma Phys. Control. Fusion 48557

[36] Fujisawa A et al 2007 Nucl. Fusion 47 S718

[37] Mertens V, Raupp G and Treutterer W 2003 Fusion Sci. Tech. 44593

[38] McCarthy P J 1999 Phys. Plasmas 63554

[39] Krämer-Flecken A, Soldatov S, Koslowski H R, Zimmermann O and TEXTOR Team 2006 Proc. 33rd EPS Conf. Plasma Phys. (Rome) ECA vol 30I (Mulhouse: EPS) P-2.152

[40] Yu C X, Brower D L, Zhao S J et al 1992 Phys. Fluids B 4381

[41] Lebedev V B, Yushmanov P N, Diamond P H, Novakovskii S V and Smolyakov A I 1996 Phys. Plasmas 33023

[42] Reich M, Wolfrum E, Schweinzer J, Ehmler H, Horton L D, Neuhauser J and ASDEX Upgrade Team 2004 Plasma Phys. Control. Fusion 46797

[43] Stringer T E 1970 Phys. Fluids 131586

[44] Itoh S-I, Itoh K, Sasaki M, Fujisawa A, Ido T and Nagashima Y 2007 Plasma Phys. Control. Fusion 49 L7 